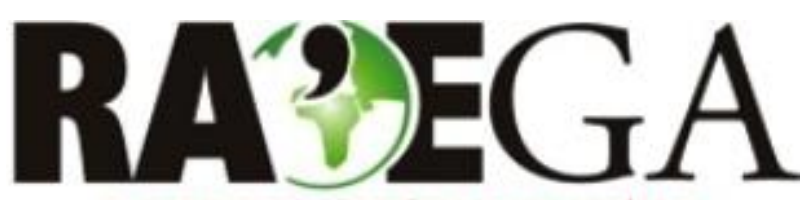

O ESPACYO GEOGRÁFICO EM ANÁLISE

\title{
ZONEAMENTO AMBIENTAL ACÚSTICO COMO ESTRATÉGIA DE GESTÃO E CONTROLE DA POLUIÇÃO SONORA URBANA
}

\section{ACOUSTIC ENVIRONMENTAL ZONING AS STRATEGY OF MANAGEMENT AND CONTROL OF THE URBAN NOISE POLLUTION}

\author{
Adriano Bressane \\ Universidade Estadual Paulista - UNESP \\ Sorocaba, SP, Brasil \\ e-mail: adrianobressane@ymail.com \\ Patricia Satie Mochizuki \\ Secretaria do Meio Ambiente \\ e-mail:patriciasm@ambiente.sp.gov.br \\ Rosana Maria Caram \\ Universidade de São Paulo - USP \\ São Carlos, SP, Brasil \\ e-mail: rcaram@sc.usp.br \\ José Arnaldo Frutuoso Roveda \\ Universidade Estadual Paulista - UNESP \\ Sorocaba, SP, Brasil \\ e-mail: roveda@sorocaba.unesp.br
}

\section{Resumo}

Os impactos da poluição sonora a tornaram uma das mais graves formas de degradação ambiental. Entretanto, considerando o constante crescimento urbano e demográfico, com respectivo aumento e adensamento das fontes de ruído, as ações de controle tornam-se tão complexas, quanto urgentes. Nesse contexto, o objetivo do estudo foi desenvolver uma metodologia para 0 zoneamento acústico como estratégia de controle, integrada ao ordenamento territorial, aplicado como estudo de caso em Rio Claro/SP. Para isso, diretrizes normativas e referências da literatura sobre a cartografia estratégica do ruído, constituíram os principais materiais utilizados. Dessa forma, foram propostos padrões e zonas acústicas associadas à tipologia de uso do solo, proteção de estabelecimentos sensíveis e prevenção de conflitos de vizinhança. Como 


\title{
ZONEAMENTO AMBIENTAL ACÚSTICO COMO ESTRATÉGIA DE GESTÃo E CONTROLE DA POLUIÇÃO SONORA URBANA
}

resultado da aplicação na área de estudo, obteve-se zonas de sensibilidade ao ruído, destinadas à: proteção da biodiversidade urbana $\left(21,0 \mathrm{~km}{ }^{2}\right)$; manutenção de condições salubres em áreas residências, escolas e hospitais $\left(8,3 \mathrm{~km}^{2}\right)$; instalação de estabelecimentos comerciais, de serviços e industriais não incômodos $\left(10,3 \mathrm{~km}^{2}\right)$; prevenção contra a exposição de moradores, trabalhadores e consumidores em áreas de uso diversificado $\left(11,2 \mathrm{~km}^{2}\right)$; condições e extensões territoriais necessárias ao desenvolvimento de atividades ruidosas $\left(9,9 \mathrm{~km}^{2}\right)$. Assim, conclui-se que o procedimento proposto para o zoneamento ambiental acústico pode contribuir com o avanço em soluções aplicáveis ao controle da poluição sonora, colaborando para o alcance de cidades saudáveis e sustentáveis.

Palavras chaves: Ruído urbano; Cartografia estratégica; Uso do solo.

\begin{abstract}
Its impacts transformed noise pollution in one of the most severe forms of environmental degradation. However, considering the constant urban and demographic growing, with respective increase and densification of noise sources, control actions become so complex, as urgent. In this context, the aim of this study was to develop a methodology to the acoustic zoning as control strategy, integrated territorial planning, applied as study case in Rio Claro city, São Paulo State, Brazil. For that, law guidelines and literature references on strategic noise mapping, constituted the main materials used. Thus, were proposed standards and acoustic zones associated with typology of land use, protection of sensitive establishments and prevention of neighborhood conflicts. As a results of application in the study area, was obtained sensitive to noise zones, destined for: protection of urban biodiversity $\left(21.0 \mathrm{~km}^{2}\right)$; maintenance of healthy conditions in residential areas, schools and hospitals $\left(8.3 \mathrm{~km}^{2}\right)$; installation of trade, services and industrial not annoying $\left(10.3 \mathrm{~km}^{2}\right)$; exposure prevention of residents, workers and consumers in areas with mixed use (11.2 $\mathrm{km}^{2}$ ); territorial conditions and extensions necessary for the development of noisy activities $\left(9.9 \mathrm{~km}^{2}\right)$. Therefore, we conclude that the procedure proposed to acoustic environmental zoning can contribute to advancement of solutions for the control of noise pollution, collaborating to achieve healthy and sustainable cities.
\end{abstract}

Keywords: Urban noise; Strategic cartography; Land use.

\section{INTRODUÇÃO}

A poluição sonora consiste no ruído excessivo, emissão e propagação de energia acústica que altera as condições ambientais em níveis superiores aos padrões estabelecidos, comprometendo a saúde humana e demais formas de vida (BRESSANE, SANTARINE e MAURICIO, 2010).

$\mathrm{Na}$ falta de controle, seus impactos a tornaram uma das mais graves 
formas de degradação urbana, com efeitos sobre o stress e qualidade de vida, humor e distúrbios comportamentais, desempenho físico e mental, hipertensão, alterações bioquímicas e hormonais (FOLSCHER et al. 2014; BASNER et al., 2014; PORTELA et al., 2013; SZEREMETA e ZANNIN, 2013; FIRDAUS, 2010; PAZ e ZANNIN, 2009; BABISCH, 2008; STANSFELD e MATHESON, 2003; ZANNIN et al., 2002).

Dessa forma, além de afetar a saúde pública, a poluição sonora ainda pode comprometer atividades socioeconômicas e a manutenção da biodiversidade urbana, ao afugentar e interferir na reprodução da fauna que atua como dispersora genética da flora (HALFWERK et al., 2011; KIGHT e SWADDLE, 2011; BARBER, CROOKS e FRISTRUP, 2011; GOINES e HAGLER, 2007).

Nesse contexto, estudos têm apontado que o mapeamento por meio de simuladores e medições sonoras é de extrema importância para caracterizar o ruído ambiental e orientar o planejamento urbano (BUNN e ZANNIN, 2015; SOUZA FILHO et al., 2015; CASAS et al., 2014; GUEDES et al., 2011).

Assim, medições do nível pressão sonora e o levantamento de parâmetros indiretos, como a contagem do fluxo de veículos, possibilitam indicadores quantitativos sobre a condição local (FIEDLER e ZANNIN, 2015a; ZANNIN e BUNN, 2014; ZANNIN e SANT'ANA, 2011).

Por sua vez, o estudo da percepção, por meio de entrevistas com a população exposta ao ruído ambiental, pode proporcionar um diagnóstico participativo, norteando ações para recuperação da qualidade sonora nos centros urbanos (SZEREMETA e ZANNIN, 2015; ENGEL et al., 2014; ZANNIN et al., 2013; ZANNIN, FERREIA e SZEREMETA, 2006; PAZ, FERREIRA e ZANNIN, 2005; ZANNIN e SZEREMETTA, 2003).Considerando o contínuo crescimento urbano, consequente aumento e adensamento das fontes de poluição sonora, o objetivo desse estudo foi desenvolver uma metodologia aplicada ao zoneamento acústico como estratégia de controle, integrada ao planejamento do uso do solo. Assim, o procedimento proposto foi aplicado como estudo de caso no município de Rio Claro/SP. 


\section{MATERIAIS E MÉTODOS}

A área de estudo corresponde à zona urbana de Rio Claro/SP (Figura 1), considerado um município de porte médio com elevado nível de riqueza e bons indicadores sociais, possui cerca de 498 km², uma população de 192 mil habitantes e situa-se a $173 \mathrm{~km}$ da capital paulista (SEADE, 2015).

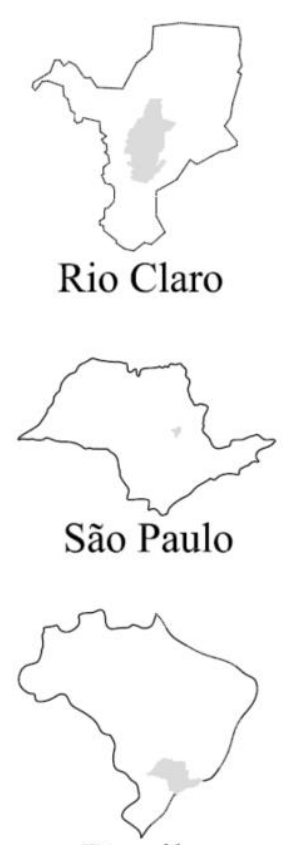

Brasil

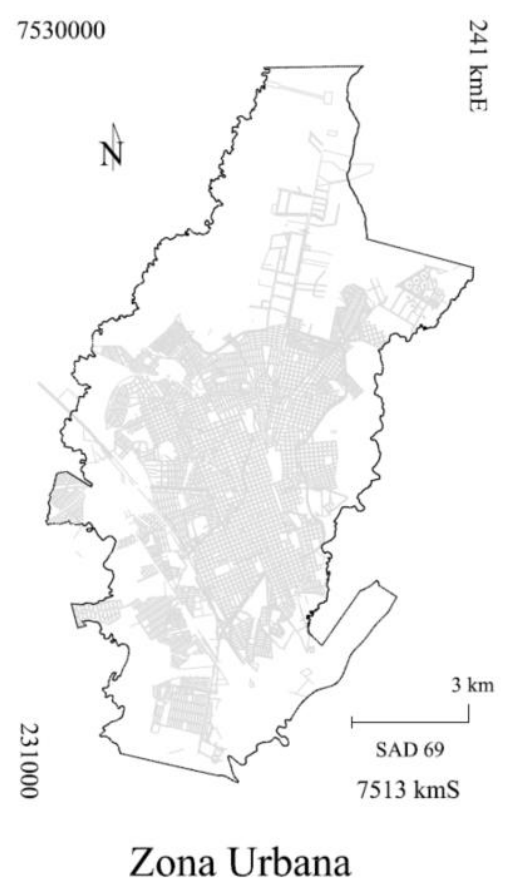

Figura 1. Localização da área de estudo, zona urbana de Rio Claro/SP.

Embora importantes para o diagnóstico do ruído ambiental, nesse estudo não foram realizadas medições sonoras, contagens de veículos, nem entrevistas com a população, pois o zoneamento proposto baseia-se em um enquadramento acústico das categorias de uso do solo, como descrito a seguir.

Para tanto, os principais materiais utilizados corresponderam às diretrizes da legislação aplicável. Dessa forma, os níveis sonoros propostos para cada zona acústica foram baseados em valores de referência estabelecidos pela Associação Brasileira de Normas Técnicas (ABNT, 2000) e a Companhia Ambiental do Estado de São Paulo (CETESB, 1992).

$A$ atribuição destes valores às zonas acústicas propostas para área de estudo considerou as diretrizes locais de uso e ocupação do solo, regulamentadas pela Lei Municipal 3806 (RIO CLARO, 2007), que constitui o 
Plano Diretor vigente.

A NBR 10.151 (ABNT, 2000) foi definida a nível federal pela Resolução Conama 01 (BRASIL, 1990), e a NL 11.032 (CETESB, 1992) em âmbito estadual paulista pelo Decreto 8.468 (SÃO PAULO, 1976), como normas que regulamentam o controle da poluição sonora em áreas habitadas.

Complementarmente, ainda foram estudadas diretrizes da Comunidade Europeia, como referência internacional no desenvolvimento de estratégias para o controle da poluição sonora, por meio da construção de mapas acústicos para territórios municipais (EUROPEAN UNION, 2002).

A base cartográfica utilizada para o zoneamento ambiental acústico da área de estudo foi obtida junto a prefeitura de Rio Claro (SP), composta pelo sistema viário, zonas de uso e ocupação do solo, localização de unidades de saúde e ensino (RIO CLARO, 2015).

Para complementar tais informações, ainda foi realizado um levantamento de hospitais, escolas e similares por meio de consultas a instituições do governo municipal, principalmente, a Secretaria Municipal de Educação e a Fundação Municipal de Saúde.

\section{RESULTADOS E DISCUSSÃO}

\subsection{Desenvolvimento da metodologia para o zoneamento ambiental acústico}

Constatando os impactos da poluição sonora, a Comunidade Europeia institucionalizou um programa global, uniforme e integrado, visando coordenar políticas e iniciativas comuns aos Estados-Membros (UNIÓN EUROPEA, 1996).

Dessa forma foi instituída a Diretiva 42 (EUROPEAN UNION, 2002) por meio da qual vem sendo desenvolvida a Cartografia Estratégica do Ruído, definida como o conjunto de estudos e ações para implantar instrumentos aplicáveis ao controle da poluição sonora integrado ao ordenamento territorial.

Entre as regulamentações dessa diretiva, destaca-se o Decreto-Lei 9 (PORTUGAL, 2007), que estabelece a delimitação espacial do território em zonas conforme sua sensibilidade ao ruído:

R. Ra'e Ga - Curitiba, v. 35, p 147 - 168 , Dez/2015 
- zonas sensíveis, muita sensibilidade ao ruído, admitindo $55 \mathrm{~dB}(\mathrm{~A})$ diurnos e $45 \mathrm{~dB}(\mathrm{~A})$ noturnos;

- zonas mista de grau I, bastante sensibilidade ao ruído, admitindo $60 \mathrm{~dB}(\mathrm{~A})$ diurnos e $50 \mathrm{~dB}(\mathrm{~A})$ noturnos; e

- zona mista de grau II, com alguma sensibilidade ao ruído, admitindo $65 \mathrm{~dB}(\mathrm{~A})$ diurnos e $60 \mathrm{~dB}(\mathrm{~A})$ noturnos.

As zonas sensíveis consistem naquelas destinadas à habitação, escolas, hospitais e espaços de lazer, existentes ou previstos. Por sua vez, as zonas mistas são as que possuem ocupação destinada para outros usos.

Como base nessas diretrizes, mapas de zoneamento acústico vêm sendo elaborados para o território de cidades europeias, com a representação da distribuição geográfica de padrões limite de nível sonoro, visando o enquadramento futuro das áreas em uma condição desejada (MATEOS, VILLALTA e ALBA, 2012; KING, MURPHY e RICE, 2011; DIAZ et al., 2010; MURPHY e KING, 2010; KING e RICE, 2009; PICCOLO, PLUTINO e CANNISTRARO, 2005).

Embora Prascevic et al. (2013) aponte a ausência de uma metodologia internacionalmente reconhecida para o zoneamento acústico, diversos estudos sobre mapeamento acústico urbano têm sido realizados no Brasil, com avanços em procedimentos de análise, envolvendo medições e simulações (FIEDLER e ZANNIN, 2015a, 2015b; BUNN e ZANNIN, 2015; SOUZA FILHO et al., 2015; ENGEL et al., 2014; ZANNIN et al., 2013; ZANNIN e SANT'ANA, 2011).

No Brasil, as normas atuais estabelecem níveis admissíveis visando a avaliação do ruído em áreas habitadas, como é o caso da NBR 10.151 de 2000 e a NL 11.032 de 1992. Entretanto, no país ainda não existem diretrizes normativas para o zoneamento acústico aplicado a territórios municipais, tornando necessários estudos que possam contribuir para esta finalidade.

Considerando os locais, horários e a natureza das atividades ruidosas, com vistas a compatibilizar seu exercício com a preservação da saúde e do sossego público, a NBR 10.151 instituiu os níveis sonoros admissíveis, apresentados na Tabela 1 (ABNT, 2000). 
Tabela 1. Padrões sonoros de referência na legislação federal.

\begin{tabular}{l|c|c}
\hline \multirow{2}{c|}{ Uso do solo } & \multicolumn{2}{c}{ Nível Sonoro } \\
\cline { 2 - 3 } & $\begin{array}{c}\text { Diurno } \\
(7-22 \mathrm{hs})\end{array}$ & $\begin{array}{c}\text { Noturno } \\
(22-7 \mathrm{hs})\end{array}$ \\
\hline Sítios e fazendas & $40 \mathrm{~dB}$ & $35 \mathrm{~dB}$ \\
\hline Estritamente residencial ou de hospitais ou de escolas & $50 \mathrm{~dB}$ & $45 \mathrm{~dB}$ \\
\hline Misto, predominantemente residencial & $55 \mathrm{~dB}$ & $50 \mathrm{~dB}$ \\
\hline Misto, com vocação comercial e administrativa & $60 \mathrm{~dB}$ & $55 \mathrm{~dB}$ \\
\hline Misto, com vocação recreacional & $65 \mathrm{~dB}$ & $55 \mathrm{~dB}$ \\
\hline Predominantemente industrial & $70 \mathrm{~dB}$ & $60 \mathrm{~dB}$ \\
\hline
\end{tabular}

Fonte: Elaborada a partir da NBR 10151 (ABNT, 2000).

Em caráter complementar, no âmbito dos municípios paulistas os valores de referência foram estabelecidos pela Norma Técnica NL 11.032 de 1992, conforme apresentado na Tabela 2 (CETESB, 1992).

Tabela 2. Padrões sonoros de referência na legislação estadual paulista.

\begin{tabular}{l|c|c|c}
\hline \multirow{2}{*}{ Uso do solo } & \multicolumn{3}{c}{ Nível Sonoro } \\
\cline { 2 - 4 } & $\begin{array}{c}\text { Diurno } \\
(7-19 \mathrm{hs})\end{array}$ & $\begin{array}{c}\text { Vespertino } \\
(19-22 \mathrm{hs})\end{array}$ & $\begin{array}{c}\text { Noturno } \\
(22-7 \mathrm{hs})\end{array}$ \\
\hline Rural & $50 \mathrm{~dB}$ & $45 \mathrm{~dB}$ & $40 \mathrm{~dB}$ \\
\hline Estritamente residencial & $50 \mathrm{~dB}$ & $45 \mathrm{~dB}$ & $40 \mathrm{~dB}$ \\
\hline Predominantemente residencial & $55 \mathrm{~dB}$ & $50 \mathrm{~dB}$ & $45 \mathrm{~dB}$ \\
\hline Diversificada (uso misto) & $60 \mathrm{~dB}$ & $55 \mathrm{~dB}$ & $50 \mathrm{~dB}$ \\
\hline Predominantemente industrial & $65 \mathrm{~dB}$ & $60 \mathrm{~dB}$ & $55 \mathrm{~dB}$ \\
\hline Estritamente industrial & $70 \mathrm{~dB}$ & $70 \mathrm{~dB}$ & $60 \mathrm{~dB}$ \\
\hline
\end{tabular}

Fonte: Elaborada a partir da NL 11.032 (CETESB, 1992).

Nota-se que a norma paulista amplia os períodos de referência, acrescentando o vespertino (19-22hs), por outro lado, a norma federal possui uma melhor distribuição entre os tipos de uso do solo, diferenciando as áreas mistas conforme sua vocação. Logo, pode-se admitir uma complementação entre tais diretrizes considerando que, tratando-se de legislação concorrentemente, o nível mais restritivo deve ser respeitado.

No âmbito dos municípios brasileiros, destaca-se a Lei 10.625 de 2002, 
da cidade de Curitiba/PR, que dispõe sobre ruídos urbanos e divide o território em zonas de uso, nas quais determinados níveis sonoros não podem ser excedidos por quaisquer atividades. Na referida norma municipal, os períodos e níveis sonoros a serem obedecidos são semelhantes aos definidos pela CETESB (Tabela 2), porém com maior detalhamento das zonas em diferentes categorias de uso do solo.

Como resultado do estudo desses referenciais, foram propostos os padrões sonoros e as zonas ambientais acústicas apresentados na Tabela 3.

Tabela 3. Padrões propostos para o zoneamento ambiental acústico.

\begin{tabular}{l|l|l|l|l}
\hline \multirow{2}{*}{$\begin{array}{c}\text { Zonas acústicas } \\
\text { (sensibilidade ao ruído) }\end{array}$} & \multicolumn{1}{|c|}{$\begin{array}{c}\text { Uso do solo } \\
\text { (atual ou previsto) }\end{array}$} & $\begin{array}{c}\text { Diurno } \\
(7-19 \mathrm{hs})\end{array}$ & $\begin{array}{c}\text { Vespertino } \\
(19-22 \mathrm{hs})\end{array}$ & $\begin{array}{l}\text { Noturno } \\
(7-22 \mathrm{hs})\end{array}$ \\
\hline Máxima (MXS) & $\begin{array}{l}\text { Rural e proteção } \\
\text { ambiental }\end{array}$ & $45 \mathrm{~dB}(\mathrm{~A})$ & $40 \mathrm{~dB}(\mathrm{~A})$ & $35 \mathrm{~dB}(\mathrm{~A})$ \\
\hline Alta (ALS) & $\begin{array}{l}\text { Estritamente } \\
\text { residencial }\end{array}$ & $50 \mathrm{~dB}(\mathrm{~A})$ & $45 \mathrm{~dB}(\mathrm{~A})$ & $40 \mathrm{~dB}(\mathrm{~A})$ \\
\hline Média a alta (MAS) & $\begin{array}{l}\text { Predominante } \\
\text { residencial }\end{array}$ & $55 \mathrm{~dB}(\mathrm{~A})$ & $50 \mathrm{~dB}(\mathrm{~A})$ & $45 \mathrm{~dB}(\mathrm{~A})$ \\
\hline Média (MDS) & $\begin{array}{l}\text { Vocação comercial } \\
\text { e administrativa }\end{array}$ & $60 \mathrm{~dB}(\mathrm{~A})$ & $55 \mathrm{~dB}(\mathrm{~A})$ & $50 \mathrm{~dB}(\mathrm{~A})$ \\
\hline Média a baixa (MBS) & $\begin{array}{l}\text { Vocação mista } \\
\text { indefinida }\end{array}$ & $60 \mathrm{~dB}(\mathrm{~A})$ & $60 \mathrm{~dB}(\mathrm{~A})$ & $55 \mathrm{~dB}(\mathrm{~A})$ \\
\hline Baixa (BXS) & $\begin{array}{l}\text { Predominante } \\
\text { industrial }\end{array}$ & $65 \mathrm{~dB}(\mathrm{~A})$ & $60 \mathrm{~dB}(\mathrm{~A})$ & $55 \mathrm{~dB}(\mathrm{~A})$ \\
\hline Mínima (MNS) & $\begin{array}{l}\text { Estritamente } \\
\text { industrial }\end{array}$ & $70 \mathrm{~dB}(\mathrm{~A})$ & $70 \mathrm{~dB}(\mathrm{~A})$ & $70 \mathrm{~dB}(\mathrm{~A})$ \\
\hline
\end{tabular}

Os padrões sonoros e zonas acústicas foram associadas a tipologia de uso e ocupação do solo, comumente observada em planos diretores municipais, constituindo uma proposta de adequação da metodologia europeia a legislação brasileira, que resultou em um maior número de classes.

Outro aspecto considerado foi a localização de equipamentos urbanos mais sensíveis ao ruído, como unidades de ensino, hospitais e similares, eventualmente situados em zonas menos sensíveis, caso em que precisam ser especialmente protegidos.

Para isso, foi proposta a criação de Zonas Sensíveis (ZS), delimitandose subáreas com um raio de 50 metros a partir do limite da quadra urbana em 
que se situam os equipamentos sensíveis, complementada por Zonas de Amortecimento Acústico (ZAA).

Considerando fronteiras entre zonas com diferentes sensibilidades ao ruído, o padrão de referência mais restritivo deve ser atendido. Contudo, para orientar o planejamento, instalação, operação e fiscalização de empreendimentos potencialmente ruidosos, torna-se importante estabelecer referências espaciais. Para isso, ZAA também foram propostas para transição gradual do nível sonoro admitido.

Em campos acústicos abertos, o nível de pressão sonora (NPS) decai cerca de $6 \mathrm{~dB}(\mathrm{~A})$ a cada duplicação da distância em relação à fonte emissora, como pode ser calculado pela equação (1) (GERGES, 2000):

$$
N P S=d B_{0}-10 \log \frac{d}{d_{0}}
$$

onde,

$d B_{0}$, nível de pressão sonora na distância $d_{0}$;

$d_{0}$, distância inicial em relação à fonte emissora;

$d$, distância final em relação à fonte emissora.

Considerando esse fenômeno, propôs-se que as ZAA avancem, progressivamente, 50 metros a partir dos limites das zonas acústicas mais restritivas sobre as zonas menos sensíveis adjacentes, visando atenuar $5 \mathrm{~dB}(\mathrm{~A}) / 50 \mathrm{~m}$. Dessa forma, reduz-se a possibilidade de conflitos de vizinhança, entretanto, apenas recorrendo aos conceitos de emissão e imissão sonora sua prevenção pode ser plenamente solucionada nas zonas de amortecimento.

Nesse sentido, definiu-se que o nível total de ruído em um dado local corresponde ao som emitido (que sai) por fontes internas a zona em que se localiza, adicionado ao ruído imitido (que entra) por fontes externas a esta mesma zona.

Assim, para as zonas de amortecimento foram definidos níveis diferenciados aos ruídos emitidos e imitidos, visando assegurar que o som proveniente da zona vizinha não extrapole o nível total admissível para a zona 
adjacente, conforme proposto na Tabela 4.

Tabela 4. Níveis admissíveis propostos para as zonas de amortecimento (ZAA), onde: D - Diurno, V - Vespertino. N - Noturno.

\begin{tabular}{l|c|c|c|c|c|c}
\hline \multirow{2}{*}{ Zonas de Amortecimento Acústico ZAA } & \multicolumn{6}{|c}{ Nível Sonoro - dB(A) } \\
\cline { 2 - 7 } & \multicolumn{2}{|c|}{ Emissão } & \multicolumn{2}{|c}{ Imissão } \\
\cline { 3 - 7 } & $\mathbf{D}$ & $\mathbf{V}$ & $\mathbf{N}$ & $\mathbf{D}$ & $\mathbf{V}$ & $\mathbf{N}$ \\
\hline ZAA de Máxima sensibilidade (ZAA-MXS) & 45 & 40 & 35 & 50 & 45 & 40 \\
\hline ZAA de Alta sensibilidade (ZAA-ALS) & 50 & 45 & 40 & 55 & 50 & 45 \\
\hline ZAA de Média a alta sensibilidade (ZAA-MAS) & 55 & 50 & 45 & 60 & 55 & 50 \\
\hline ZAA de Média sensibilidade (ZAA-MDS) & 60 & 55 & 50 & 65 & 60 & 55 \\
\hline ZAA de Média a Baixa sensibilidade (ZAA-MBS) & 60 & 60 & 55 & 65 & 65 & 60 \\
\hline ZAA de Baixa sensibilidade (ZAA-BXS) & 65 & 60 & 55 & 70 & 65 & 60 \\
\hline
\end{tabular}

Para orientar a criação das zonas de amortecimento, na Tabela 5 é apresentada a variação entre níveis sonoros admissíveis em zonas adjacentes nos diferentes períodos, diurno, vespertino e noturno, devendo ser considerada a máxima variação existente em cada caso.

Tabela 5. Variação do nível sonoro em zonas adjacentes em dB(A).

\begin{tabular}{|c|c|c|c|c|c|c|c|c|c|c|c|c|c|c|c|c|c|c|c|c|c|}
\hline & \multicolumn{3}{|c|}{ MXS } & \multicolumn{3}{|c|}{ ALS } & \multicolumn{3}{|c|}{ MAS } & \multicolumn{3}{|c|}{ MDS } & \multicolumn{3}{|c|}{ MBS } & \multicolumn{3}{|c|}{ BXS } & \multicolumn{3}{|c|}{ MNS } \\
\hline & D & $\mathbf{V}$ & $\mathbf{N}$ & D & V & $\mathbf{N}$ & D & $\mathbf{V}$ & $\mathbf{N}$ & D & V & $\mathbf{N}$ & D & V & $\mathbf{N}$ & D & V & $\mathbf{N}$ & D & V & $\mathbf{N}$ \\
\hline MXS & 0 & 0 & 0 & & & & & & & & & & & & & & & & & & \\
\hline ALS & 5 & 5 & 5 & 0 & 0 & 0 & & & & & & & & & & & & & & & \\
\hline MAS & 10 & 10 & 10 & 5 & 5 & 5 & 0 & 0 & 0 & & & & & & & & & & & & \\
\hline MDS & 15 & 15 & 15 & 10 & 10 & 10 & 5 & 5 & 5 & 0 & 0 & 0 & & & & & & & & & \\
\hline MBS & 15 & 20 & 20 & 10 & 15 & 15 & 5 & 5 & 5 & 0 & 5 & 5 & 0 & 0 & 0 & & & & & & \\
\hline BXS & 20 & 20 & 20 & 15 & 15 & 15 & 10 & 10 & 10 & 5 & 5 & 5 & 5 & 0 & 0 & 0 & 0 & 0 & & & \\
\hline MNS & 25 & 30 & 35 & 20 & 25 & 30 & 15 & 15 & 15 & 10 & 15 & 20 & 10 & 10 & 15 & 5 & 10 & 15 & 0 & 0 & 0 \\
\hline
\end{tabular}

Pela tabela anterior, nota-se que o nível sonoro pode variar significativamente de 5 a $35 \mathrm{~dB}(\mathrm{~A})$ entre zonas adjacentes, destacando a importância das zonas de amortecimento para transição gradual e controle de atividades potencialmente ruidosas instaladas próximas ao limite entre zonas.

Por fim, com a finalidade de integrar o controle da poluição sonora ao 
ordenamento territorial, destaca-se:

- o procedimento proposto para o zoneamento acústico visa a comunicação visual da distribuição geográfica dos níveis sonoros máximos admissíveis;

- o zoneamento acústico proposto consiste numa prognóstico do cenário futuro desejado, logo, não substitui a necessidade das medições sonoras, que são de extrema importância para o diagnóstico da condição sonora existente;

- o objeto de estudo ou área de aplicação corresponde ao território municipal, mas pode destinar-se apenas ao perímetro urbano ou regiões de interesse; e

- recomenda-se que a representação cartográfica na forma impressa deve possuir escala capaz de permitir a identificação das quadras e sistema viário que integra cada zona.

\subsection{Zoneamento ambiental acústico da área urbana de Rio Claro (SP)}

A metodologia proposta para o zoneamento ambiental acústico foi aplicada como estudo de caso na área urbana de Rio Claro/SP, que totaliza cerca de $80 \mathrm{~km}^{2}$, o equivalente a 16\% do município (RIO CLARO, 2007), por meio de três etapas principais:

- delimitação espacial das zonas acústicas mediante o estudo das vocações e restrições de uso do solo previstas na legislação vigente;

- identificação de equipamentos urbanos equivalentes a escolas, hospitais e similares para criação de zonas sensíveis no entorno; e

- criação das zonas de amortecimento correspondentes a faixas de transição gradual entre zonas adjacentes.

Conforme a Lei Municipal 3806 (RIO CLARO, 2007), são previstas as categorias de uso urbano residencial $(R)$, comercial $(C)$, serviços $(S)$, industrial (I), institucional $(E)$ e proteção ambiental $(P)$, apresentadas na Tabela 6, que podem ser classificadas como:

- uso conforme, permitido para uma determinada zona (vocação);

- uso controlado, permitido em determinadas condições urbanísticas; e

- uso não conforme, vedado (restrição plena). 
Tabela 6. Categorias de uso do solo na área urbana de Rio Claro (SP).

\begin{tabular}{|c|c|}
\hline Categoria de uso do solo & $\begin{array}{l}\text { Principais características relacionadas à } \\
\text { impactos por ruídos excessivos }\end{array}$ \\
\hline Residencial unidomiciliar (R1) & $\begin{array}{l}\text { Edificações para habitação permanente, com } \\
\text { apenas um domicílio por lote }\end{array}$ \\
\hline Residencial multidomiciliar (R2) & $\begin{array}{l}\text { Idem a R1, mas com mais de um domicílio } \\
\text { por lote }\end{array}$ \\
\hline Comércio varejista local (C1) & $\begin{array}{l}\text { Estabelecimentos não ruidosos, de venda } \\
\text { direta ao consumo doméstico }\end{array}$ \\
\hline Comércio varejista diversificado (C2) & $\begin{array}{l}\text { Estabelecimentos de venda direta de } \\
\text { produtos }\end{array}$ \\
\hline Comé & $\begin{array}{l}\text { Comércio não varejista de produtos } \\
\text { relacionados ou não ao uso residencial }\end{array}$ \\
\hline Servi & $\begin{array}{l}\text { Destinado à prestação de serviços } \\
\text { compatível com padrões de uso residencial }\end{array}$ \\
\hline Serviço diversi & \begin{tabular}{|lll} 
Destinado à prestação de serviços \\
potencialmente ruidosos
\end{tabular} \\
\hline Serviç & $\begin{array}{l}\text { Estabelecimentos de prestação de serviços, } \\
\text { especialmente incômodos }\end{array}$ \\
\hline Indústrias não incômodas (I1) & $\begin{array}{l}\text { Processos que se adéquam aos mesmos } \\
\text { padrões de uso não industrial }\end{array}$ \\
\hline Indústr & $\begin{array}{l}\text { Atividades que necessitam de métodos } \\
\text { especiais de controle ambiental }\end{array}$ \\
\hline Indústrias especiais (I3) & $\begin{array}{l}\text { Atividades capazes de impactar à saúde e } \\
\text { segurança da população, mesmo controladas }\end{array}$ \\
\hline Institucional diversific & $\begin{array}{l}\text { Destinadas à saúde, educação, cultura, lazer } \\
\text { ou administração pública, não ruidosas }\end{array}$ \\
\hline Institucional especial (E2) & $\begin{array}{l}\text { Idem, mas com características que implicam } \\
\text { em medidas especiais pelo poder público }\end{array}$ \\
\hline Institucional i & $\begin{array}{l}\text { Outras instituições com impactos que exigem } \\
\text { medidas especiais pelo poder público }\end{array}$ \\
\hline Proteç & $\begin{array}{l}\text { Áreas com finalidade de proteção de } \\
\text { recursos ambientais e da biodiversidade }\end{array}$ \\
\hline
\end{tabular}

Fonte: Elaborada a partir da Lei Municipal 3806 (RIO CLARO, 2007).

Em contraste a Lei 10.625 de 2002, de Curitiba/PR, a Lei 3806 de 2007, de Rio Claro/SP não estabelece os níveis sonoros admissíveis para as zonas com diferentes categoria de uso do solo. Assim, como resultado do estudo das características apresentadas, as diferentes categorias de uso que integram a área urbana de Rio Claro/SP foram enquadradas, considerando sua conformidade (vocação e restrição), nas zonas acústicas propostas na Tabela 7. 
BRESSANE, A.; MOCHIZUKI, P. S.; CARAM, R. M.; ROVEDA, J. A. F.

ZONEAMENTO AMBIENTAL ACÚSTICO COMO ESTRATÉGIA DE GESTÃO E CONTROLE DA POLUIÇÃO SONORA URBANA

Tabela 7. Enquadramento das categorias de uso do solo nas zonas acústicas.

\begin{tabular}{l|l|l}
\hline \multirow{2}{*}{ Zonas acústicas } & \multicolumn{2}{c}{ Categorias de uso do solo } \\
\cline { 2 - 3 } & \multicolumn{1}{c}{ Conforme } & \multicolumn{1}{c}{ Controlado } \\
\hline Máxima sensibilidade (MXS) & $\mathrm{R} 1, \mathrm{P}, \mathrm{E} 1$ & $\mathrm{R} 2$ \\
\hline Alta sensibilidade (ALS) & $\mathrm{R} 1, \mathrm{E} 1$ & $\mathrm{R} 2, \mathrm{E} 2$ \\
\hline Média a alta sensibilidade (MAS) & $\mathrm{R} 1, \mathrm{R} 2$ & $\mathrm{C} 1, \mathrm{C} 2, \mathrm{~S} 1, \mathrm{~S} 2, \mathrm{E} 2, \mathrm{I}$ \\
\hline Média sensibilidade (MDS) & $\mathrm{R} 1, \mathrm{R} 2, \mathrm{C} 1, \mathrm{~S} 1$ & $\mathrm{C} 2, \mathrm{C} 3, \mathrm{~S} 2, \mathrm{~S} 3, \mathrm{E} 2, \mathrm{I1}$ \\
\hline Média a baixa sensibilidade (MBS) & $\mathrm{R} 1, \mathrm{~S} 1, \mathrm{C} 1, \mathrm{E} 2$ & $\mathrm{R} 2, \mathrm{C} 2, \mathrm{C} 3, \mathrm{~S} 2, \mathrm{~S} 3, \mathrm{E} 3$ \\
\hline Baixa sensibilidade (BXS) & $\mathrm{C}, \mathrm{S}, \mathrm{I1}, \mathrm{I}, \mathrm{E} 2$ & $\mathrm{R} 2, \mathrm{I}, \mathrm{E} 3$ \\
\hline Mínima sensibilidade (MNS) & $\mathrm{I}, \mathrm{I} 2, \mathrm{I} 3$ & $\mathrm{E}, \mathrm{S}, \mathrm{C}$ \\
\hline
\end{tabular}

Como resultado do levantamento para complementação da base cartográfica foram totalizados 88 equipamentos urbanos sensíveis ao ruído na área de estudo, sendo 24 unidades de saúde e 64 unidades escolares. A construção das Zonas Sensíveis (ZS) e de Amortecimento (ZAA) para proteção especial destes equipamentos pode ser observada na Figura 2.

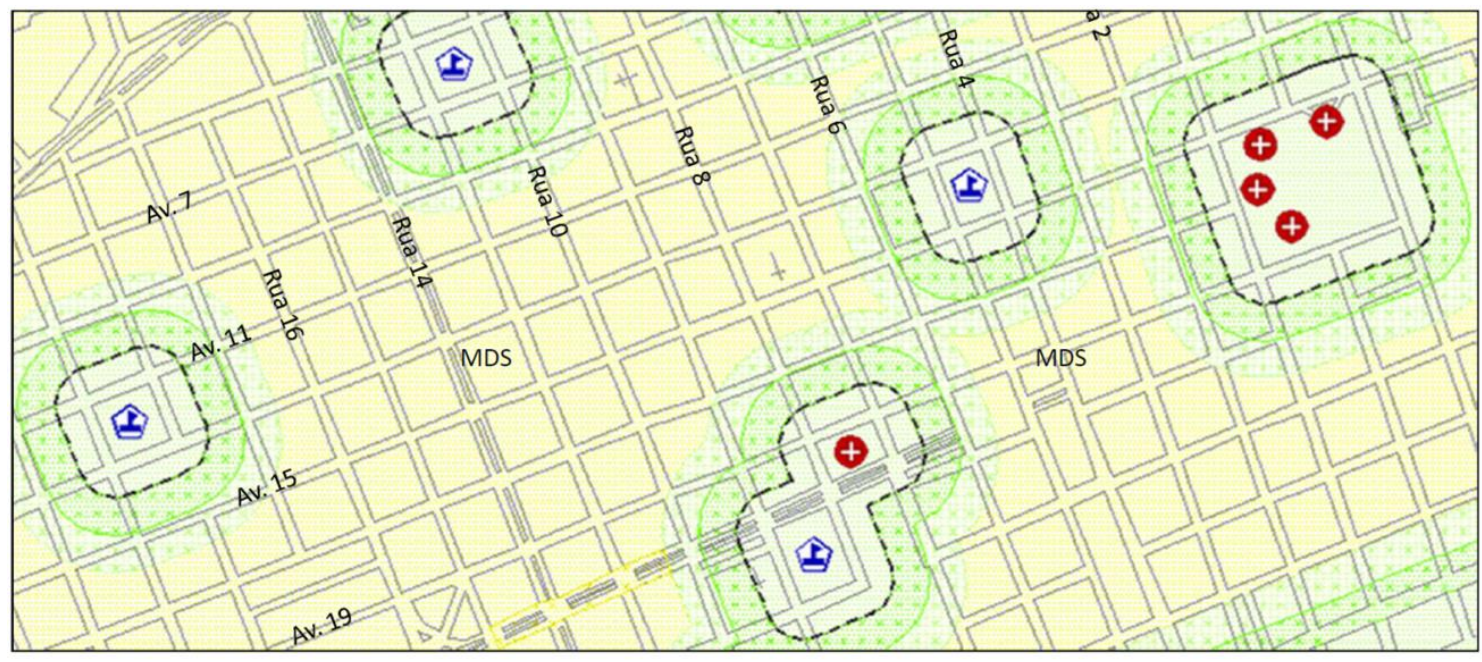

Zonas Acústicas

Máxima Sensibilidade
Alta Sensibilidade
Média Alta Sensibilidade
Média Sensibilidade
Média Baixa Sensibilidade
Baixa Sensibilidade
Mínima Sensibilidade

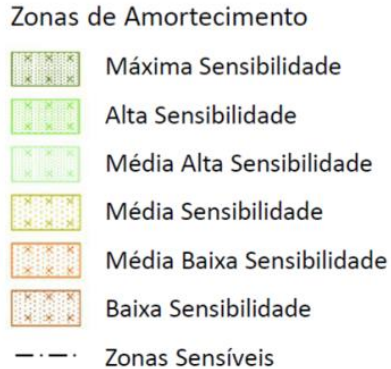

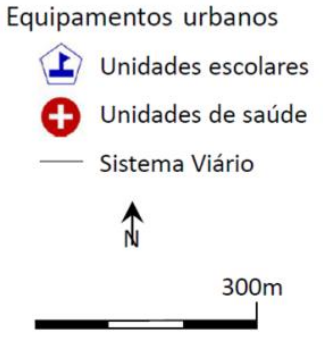

ntos urbanos

nidades escolares

+ Unidades de saúde

- Sistema Viário

Figura 2. Detalhe da delimitação espacial das Zonas Sensíveis e das ZAA. 
A geração das zonas de amortecimento para transição gradual entre zonas acústicas adjacentes pode ser visualizada na Figura 3, na qual destacase que as faixas foram delimitadas partindo do limite entre zonas e avançando sobre aquelas com menor sensibilidade.
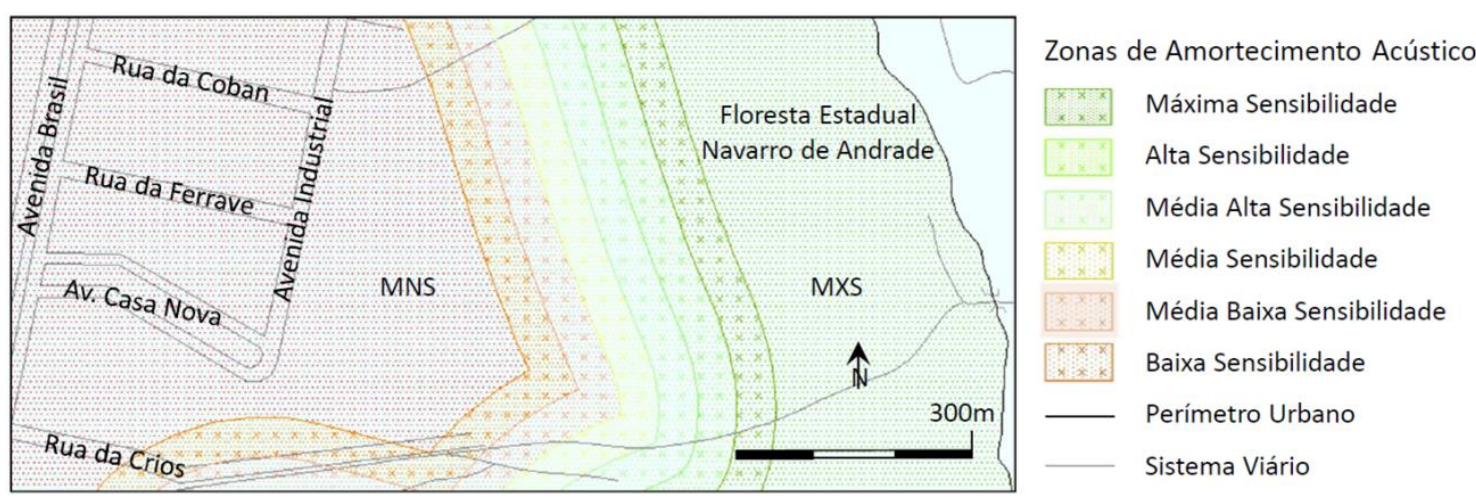

Figura 3. Detalhe da delimitação espacial das ZAA.

Dessa forma, a aplicação da metodologia proposta na área de estudo resultou no zoneamento ambiental acústico urbano de Rio Claro (SP), apresentado na Figura 4, na qual foi utilizado um sistema alternativo de cores nas hachuras para um melhor contraste visual em função da escala.

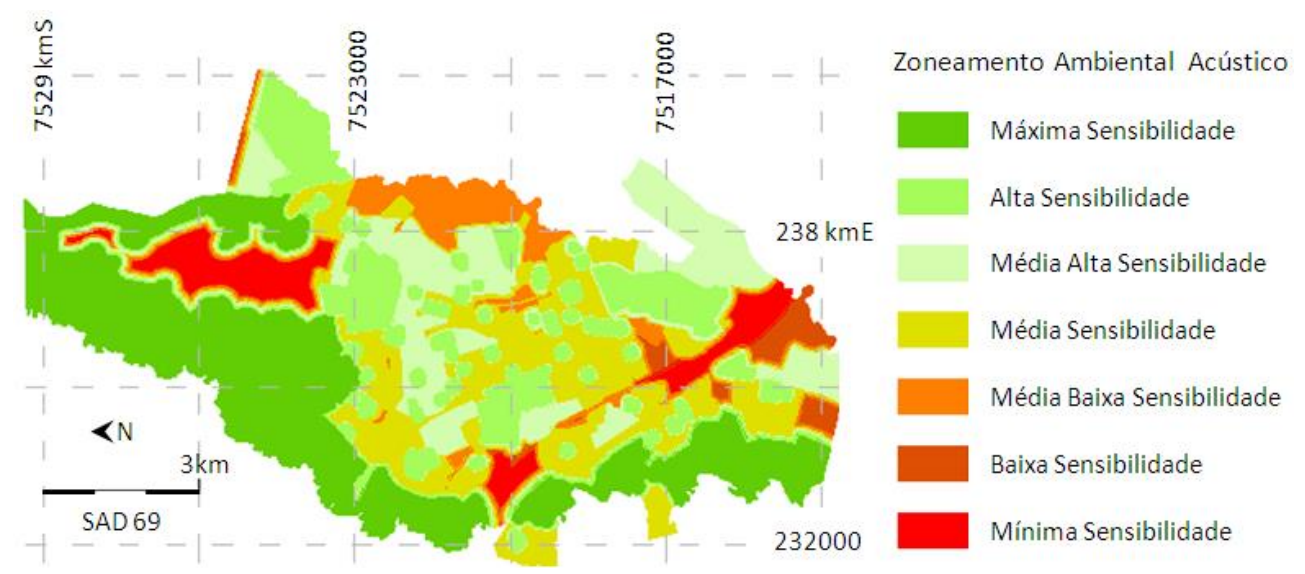

Figura 4. Zoneamento ambiental acústico da área urbana de Rio Claro (SP).

A zona de máxima sensibilidade ao ruído (MXS) abrangeu 21,03 km², 0 equivalente a $26,3 \%$ da área urbana. Apesar de admitir uso residencial unifamiliar, escolas e hospitais, esta zona destina-se principalmente a proteção 
da biodiversidade urbana contra os impactos da poluição sonora.

Composta por áreas com uso estritamente residencial, a zona de alta sensibilidade (ALS) totalizou 8,29 km². Contudo, 23,8\% dessa área também é integrada por Zonas Sensíveis destinadas a proteção de escolas e hospitais situados em zonas com níveis sonoros menos restritivos, o que representa $98,9 \%$ dessas unidades e torna evidente a importância de tais zonas.

Com uso predominantemente residencial, mas que admite de forma controlada estabelecimentos comerciais, a prestação de serviços, instalações institucionais e industriais não incômodos, a zona de média alta sensibilidade (MAS) ocupa 10,3 km², ou 13,5\% da área urbana de Rio Claro/SP.

Caracterizada pelo uso fortemente diversificado, a zona acústica de média sensibilidade (MDS) corresponde a $11,15 \mathrm{~km}^{2}$, visando prevenir a exposição ao ruído excessivo de residentes, trabalhadores e consumidores relacionados ao comércio, serviços e indústrias instaladas na área.

As áreas com vocação predominantemente industrial e para prestação de serviços ruidosos totalizaram 9,97 km², visando assegurar as condições e extensões territoriais necessárias ao desenvolvimento destas atividades. Para isso, essa extensão foi integrada pelas zonas de mínima (MNS), baixa (BXS) e média baixa (MBS) sensibilidade ao ruído, que abrangeram 4,15 km², 1,92 km² e $3,90 \mathrm{~km}^{2}$, respectivamente.

Dessa forma, as zonas ambientais acústicas totalizaram 60,74 km², complementadas por $19,26 \mathrm{~km}^{2}$ de zonas de amortecimento, responsáveis por prevenir conflitos de vizinhança entre zonas com diferentes sensibilidades ao ruído. Contudo, destaca-se que tais zonas (ZAA) possuem vocações e restrições de uso equivalentes as demais com mesma tolerância, ou equivalência de níveis sonoros admitidos.

\section{CONSIDERAÇÕES FINAIS}

Pelos resultados apresentados, considera-se que o zoneamento ambiental acústico pode constituir uma ferramenta de gestão capaz de integrar o controle da poluição sonora ao ordenamento territorial, tal como demonstrado por meio de sua aplicação na área de estudo. 
Dessa forma, considerando o fator ruído como um parâmetro urbanístico, o zoneamento acústico pode orientar a concessão ou rejeição de licenças ambientais, autorização e fiscalização de atividades potencialmente ruidosas.

Com a finalidade de proteger contra a exposição ao ruído excessivo e, ao mesmo tempo, assegurar condições necessárias a atividades sociais e econômicas, o zoneamento acústico representa uma ferramenta que favorece o acesso público à informação. Nesse sentido, apoia a gestão de obras e empreendimentos, tanto de interesse privado, como coletivo, fornecendo uma base objetiva para planejamentos setoriais, como a seleção de locais adequados para determinados empreendimentos, instituições e sistema viário.

Em conjunto com o mapeamento para diagnóstico da condição sonora existente, o zoneamento acústico possibilita avaliar a conformidade, identificar conflitos e situações críticas, favorecendo planos e metas para o gerenciamento do ruído no meio urbano.

Logo, na ausência de diretrizes legais para o zoneamento ambiental acústico no Brasil, acredita-se que os resultados desse estudo podem contribuir com o avanço em soluções aplicáveis ao controle da poluição sonora, colaborando para o alcance de cidades saudáveis e sustentáveis.

\section{REFERÊNCIAS}

ABNT. ASSOCIAÇÃO BRASILEIRA DE NORMAS TÉCNICAS. Avaliação do ruído em áreas habitadas visando o conforto da comunidade. Rio de Janeiro: ABNT, 2000.

BABISCH, W. Traffic noise and cardiovascular risk. Noise Health, London, v. 10, n. 38, p. 27-34, 2008. 
BARBER, J.R.; CROOKS, K.R.; FRISTRUP, K.M. The costs of chronic noise exposure for terrestrial organisms. Trends in Ecology e Evolution, Maryland, v. 25 , p. $180-189,2010$.

BASNER, M.; BABISCH, W.; DAVIS, A.; BRINK, M.; CLARK, C.; JANSSEN, S.; STANSFELD, S. Auditory and non-auditory effects of noise on health. The Lancet, London, v. 383, n. 9925, p. 1325-1333, 2014.

BRASIL. CONSELHO NACIONAL DO MEIO AMBIENTE. Resolução 01 de 1990. Disponível em: http://www.mma.gov.br/port/conama/res/res90 /res0190.html. Acesso em: 07 de janeiro de 2015.

BRESSANE, A.; SANTARINE, G.A.; MAURICIO, J.C. Análise fenomenológica da poluição sonora: síntese de princípios fundamentais da teoria acústica. Holos Environment, Rio Claro, v. 10, n. 2, p. 223-237, 2010.

BUNN, F.; ZANNIN, P.H.T. Urban planning - Simulation of noise control measures. Noise Control Engineering Journal, Reston, v. 63, n. 1, p. 1-10, 2015.

CASAS, W.J.P.; CORDEIRO, E.P.; MELLO, T.C.; ZANNIN, P.H.T. Noise mapping as a tool for controlling industrial noise pollution. Journal of Scientific \& Industrial Research, New Delhi, v. 73, n. 4, p. 262-266, 2014.

CETESB. COMPANHIA AMBIENTAL DO ESTADO DE SÃO PAULO. Determinação do nível de ruído em ambientes internos e externos de áreas habitadas - método de ensaio. São Paulo: CTESB, 1992.

CURITIBA (Cidade). Lei Municipal 10625 de 2002. Disponível em: http://cmcuritiba.jusbrasil.com.br/legislacao/340832/lei-10625-02. Acesso em: 10 de 
agosto de 2015.

DIAZ, J.S.; GARBÍ, J.R.; GÓMEZ, T.P.; TORRENT, M.M. Mapas estratégicos de ruido en Catalunya. Tecniacústica, León, v. 6, p. 1-8, 2010.

ENGEL, M.S.; SEGUNDO, E.H.V.; HOCHSTEINER, E.; ZANNIN, P.H.T. Statistical analysis of a combination of objective and subjective environmental noise data using factor analysis and multinomial logistic regression. Stochastic Environmental Research and Risk Assessment, Berlin, v. 28, p. 393-399, 2014.

EUROPEAN UNION. The Environmental Noise Directive. 2002. Disponível em: http://eur-lex.europa.eu/legal-content/EN/TXT/?uri=CELEX:32002L0049. Acesso em: 07 de janeiro de 2015.

FIEDLER, P.E.K.; ZANNIN, P.H.T. Evaluation of noise pollution in urban traffic hubs - Noise maps and measurements. Environmental Impact Assessment Review, Norwich, v. 51, p. 1-9, $2015 a$.

. Noise mapping as a tool for urban planing. Journal of Scientific \& Industrial Research, New Delhi, v. 74, n. 2, p. 114-116, 2015b.

FIRDAUS, G. Noise Pollution and Human Health: A Case Study of Municipal Corporation of Delhi. Indoor and Built Environment, London, v. 19, n. 6, p. 648-657, 2010.

FOLSCHER, L.L.; GOLDSTEIN, L.N.; WELLS, M.; REES, D. Emergency department noise: mental activation or mental stress? Emergency Medicine Journal, London, v. 31, n. 7, p. 1-10, 2014.

GERGES, S. N. Y. Ruído: fundamentos e controle. 2ed. Florianópolis: NR, 2000. 
GOINES, R,N.L.; HAGLER, M.D.L. Noise Pollution: A Modern Plague. Southern Medical Journal, Birmingham, v. 100, n.3, p. 287-294, 2007.

GUEDES, I.C.M.; BERTOLI, S.R.; ZANNIN, P.H.T. Influence of urban shapes on environmental noise: A case study in Aracaju Brazil. Science of the Total Environment, Barcelona, v. 412, p. 66-76, 2011.

HALFWERK, W.; HOLLEMANN, L.J.M.; LESSELLS, C.M.; SLABBEKOORN, H. Negative impact of traffic noise on avian reproductive success. Journal of Applied Ecology, London, v. 48, p. 210-219, 2011.

KIGHT, C.R.; SWADDLE, J.P. How and why environmental noise impacts animals: an integrative, mechanistic review. Ecology Letters, Montpellier v. 14, p. 1052-1061, 2011.

KING, E.A.; RICE, H.J. The development of a practical framework for strategic noise mapping. Applied Acoustics, Amsterdam, v. 70, n. 8, p. 1116-1127, 2010.

KING, E.A.; MURPHY, E.; RICE, H.J. Implementation of the EU environmental noise directive: Lessons from the first phase of strategic noise mapping and action planning in Ireland. Journal of Environmental Management, Berkeley, v. 92, n. 3, p. 756-764, 2011.

MATEOS, B.P.; VILLALTA, I.V.; ALBA, M.A. Actualización curricular y docente mediante talleres prácticos: la experiencia del taller de elaboración de mapas de contaminación acústica. Serie Geográfica, Sevilla, v. 18, p. 89-99, 2012.

MURPHY, E.; KING, E.A. Strategic environmental noise mapping: Methodological issues concerning the implementation of the EU Environmental 
Noise Directive and their policy implications. Environment International, Lancaster, v. 36, n. 3, p. 290-2098, 2010.

PAZ, E.C.; FERREIRA, A.M.C.; ZANNIN, P.H.T. Estudo comparativo da percepção do ruído urbano. Revista de Saúde Pública, São Paulo, v. 39, n. 3, p. $467-472,2005$.

PAZ, E.C.; ZANNIN, P.H.T. Avaliação do impacto ambiental prévio: poluição sonora. Revista Pavimentação, Rio de Janeiro, v. 14, p. 61-70, 2009.

PICCOLO, A.; PLUTINO, D.; CANNISTRARO, G. Evaluation and analysis of the environmental noise of Messina, Italy. Applied Acoustics, Amsterdam, v. 66 , n. 4, p. 447-465, 2005

PORTELA, B.S.; QUEIROGA, M.R.; CONSTANTINI, A.; ZANNIN, P.H.T. Annoyance evaluation and the effect of noise on the health of bus drivers. Noise Health, London, v. 15, n. 66, p. 301-306, 2013.

PORTUGAL. MINISTÉRIO DO AMBIENTE. Decreto-Lei 9 de 2007. Disponível em: http://www.dre.pt/pdf1sdip/2007/01/01200/03890398.pdf. Acesso em: 14 de janeiro de 2015.

PRASCEVIC, M.; MIHAJLOV, D.; CVETKOVIC, D.; GAJICKI, A.; HOLECEK, N. Acoustic zoning and noise assessment. Applied Mechanics and Materials, Pfaffikon, v. 430, p. 244-250, 2013.

RIO CLARO (Cidade). Base cartográfica do Município de Rio Claro/SP. Disponível em: http://www.rioclaro.sp.gov.br/pd/. Acesso em: 14 de janeiro de 2015.

Lei Municipal 3806 de 2007. Disponível em: http://www.rioclaro.sp.gov.br/diariooficial/arquivos/oficial255.pdf. Acesso em: 10 
de janeiro de 2015.

SÃO PAULO (Estado). Decreto 8.468 de 1976. Disponível em: http://www.al.sp.gov.br/repositorio/legislacao/decreto/1976/decreto-846808.09.1976.html. Acesso em: 6 de janeiro de 2015.

SEADE. FUNDAÇÃO SISTEMA ESTADUAL DE ANÁLISE DE DADOS. Perfil Municipal. Disponível em: http://produtos.seade.gov.br/produtos/perfil/perfil MunEstado.php. Acesso em: 06 de janeiro de 2015.

SOUZA FILHO, J.J.; STEFFEN, J.L.; ANDREASI, W.A.; ZANNIN, P.H.T. urban noise assessment based on noise mapping and measurements. Canadian Acoustics, Ottawa, v. 43, n. 1, p. 3-10, 2015.

STANSFELD, S.A.; MATHESON, M.P. Noise pollution: non-auditory effects on health. British Medical Bulletin, Oxford, v. 68, n. 1, p. 243-258, 2003.

SZEREMETA, B.; ZANNIN, P.H.T. A importância dos parques urbanos e áreas verdes na promoção da qualidade de vida em cidades. RA'E GA: o Espaço Geográfico em Análise, Curitiba, v. 29, p. 177-193, 2013.

A percepção dos praticantes de atividade física sobre a qualidade ambiental sonora dos parques públicos de Curitiba-Paraná. RA'E GA: o Espaço Geográfico em Análise, Curitiba, v. 33, p. 7-43, 2015.

UNIÓN EUROPEA. Política futura de lucha contra el ruido. Bruselas: Comision Europea, 1996.

ZANNIN, P.H.T.; ENGEL,M.S.; FIEDLER, P.E.K.; BUNN, F. Characterization of environmental noise based on noise measurements, noise mapping and interviews: a case study at a university campus in Brazil. Cities, Tacoma, v. 31, p. 317-327, 2013. 
ZANNIN, P.H.T.; FERREIA, A.M.C.; SZEREMETA, B. Evaluation of the noise pollution in urban parks of Curitiba. Environmental Monitoring and Assessment, Umeå, v. 118, p. 423-433, 2006.

ZANNIN, P.H.T.; BUNN, F. Noise annoyance through railway traffic - A case study. Journal of Environmental Health Science and Engineering, London, v. 12 , n. 1, p. 12-14, 2014.

ZANNIN, P.H.T.; CALIXTO, A.; DINIZ, F.B.; FERREIRA, J.A. Incômodo causado pelo ruído urbano à população de Curitiba, PR. Revista de Saúde Pública, São Paulo, v. 36, n.4, p. 521-524, 2002.

ZANNIN, P.H.T.; ENGEL, M.S.; FIEDLER, P.E.K.; BUNN, F. Characterization of environmental noise based on noise measurements, noise mapping and interviews: a case study at a university campus in Brazil. Cities, Tacoma, v. 31, p. 317-327, 2013.

ZANNIN, P.H.T.; SANT'ANA, D.Q. Noise mapping at different stages of a freeway redevelopment project A case study in Brazil. Applied Acoustics, Amsterdam, v. 72, n. 8, p. 479-486, 2011.

ZANNIN, P.H.T.; SZEREMETTA, B. Avaliação da poluição sonora no parque Jardim Botânico de Curitiba, Paraná, Brasil. Cadernos de Saúde Pública, Rio de Janeiro, v. 19, n.2, p. 683-686, 2003. 